Sugar sensing by ChREBP/Mondo-Mlx - new insight into downstream regulatory networks and integration of nutrient-derived signals

\title{
Havula, Essi
}

2018-04

Havula, E \& Hietakangas, V 2018 , ' Sugar sensing by ChREBP/Mondo-Mlx - new insight into downstream regulatory networks and integration of nutrient-derived signals ' , Current Opinion in Cell Biology , vol. 51 , pp. 89-96 . https://doi.org/10.1016/j.ceb.2017.12.007

http://hdl.handle.net/10138/311367

https://doi.org/10.1016/j.ceb.2017.12.007

cc_by_nc_nd

acceptedVersion

Downloaded from Helda, University of Helsinki institutional repository.

This is an electronic reprint of the original article.

This reprint may differ from the original in pagination and typographic detail.

Please cite the original version. 
7 1. Charles Perkins Centre, School of Life and Environmental Sciences, The

8 University of Sydney, Sydney, NSW 2006, Australia

9 2. Faculty of Biological and Environmental Sciences, University of Helsinki, Finland.

10 3. Institute of Biotechnology, University of Helsinki, Finland.

$11 *$ Correspondence: ville.hietakangas@helsinki.fi

12

13 Short title: ChREBP/Mondo-Mlx in sugar sensing

14

15 Body text word count: 3090

16

17 


\section{Highlights}

19 -ChREBP/Mondo-Mlx is an intracellular sugar sensor of animals with conserved target 20 genes and physiological functions

21 - ChREBP/Mondo-Mlx integrates multiple inputs through direct sensing of sugar and

22 fatty acid-derived metabolites and cross-talk with other nutrient sensing pathways

23 -ChREBP/Mondo-Mlx controls several second-tier regulators, including transcription 24 factors and hormones

Abstract

Animals regulate their physiology with respect to nutrient status, which requires nutrient sensing pathways. Simple carbohydrates, sugars, are sensed by the basic-helixloop-helix leucine zipper transcription factors ChREBP/Mondo, together with their heterodimerization partner Mlx, which are well-established activators of sugar-induced lipogenesis. Loss of ChREBP/Mondo-Mlx in mouse and Drosophila leads to sugar intolerance, i.e. inability to survive on sugar containing diet. Recent evidence has revealed that ChREBP/Mondo-Mlx responds to sugar and fatty acid-derived metabolites through several mechanisms and cross-connects with other nutrient sensing pathways. ChREBP/Mondo-Mlx controls several downstream transcription factors and hormones, which mediate not only readjustment of metabolic pathways, but also control feeding behavior, intestinal digestion, and circadian rhythm.

\section{Preface}

41 Nutrient intake of animals displays a high degree of variation, which requires fine-tuned control of metabolic pathways. Animals regulate their metabolic homeostasis by continuously reading and converging signals originating from both within the organism and the outside environment. Macronutrients (sugars, amino acids and lipids) are sensed by nutrient sensing pathways that detect different dietary inputs and orchestrate an integrated adaptive response that is appropriate for that particular set of macronutrients. Simple carbohydrates, sugars, are sensed by several mechanisms, of which the so-called intracellular sugar sensing by ChREBP/Mondo-Mlx transcription factors is among the most conserved ones. Studies from mouse and Drosophila have shown the importance

50 of this system in providing sugar tolerance; animals lacking ChREBP/Mondo-Mlx die

51 rapidly on a diet containing sugars at a level, which is normally physiologically 
tolerated [1,2]. We highlight here recent work, which has revealed several novel aspects of the ChREBP/Mondo-Mlx physiological function and regulation by nutrient-derived cues.

\section{ChREBP/Mondo-Mlx - the conserved regulator of metabolic homeostasis}

Carbohydrate response element binding protein (ChREBP, also known as MondoB and MLXIPL) and its paralog MondoA (MLXIP) are intracellular sugar sensors that are activated by glucose-6-phosphate (G6P) and other phosphorylated hexoses [3]. ChREBP and MondoA act together with their common heterodimerization partner Mlx, a necessary step for transcriptional activation. Although both are ubiquitously expressed, ChREBP is more prominently expressed in the liver and adipose tissue whereas MondoA is most highly expressed in the skeletal muscle. Muscle breaks down glucose via the glycolytic pathway to release energy that is used for muscle contraction and in fact many of the MondoA-Mlx target genes are involved in the glycolytic pathway [4,5]. Liver is the main site for de novo lipogenesis (DNL), where dietary sugars are converted into triacylglycerols (TAGs) and further transported to adipose tissue for storage. ChREBP target genes include lipogenic genes, such as fatty acid synthase and acetyl-CoA-carboxylase, both of which possess well-established ChREBP-Mlx binding sites, known as the carbohydrate response element (ChoRE), in their promoters [6,7]. ChREBP-Mlx is also a key regulator of glycolytic genes, such as glucokinase regulatory protein, glyceraldehyde-3-phosphate dehydrogenase and Ltype pyruvate kinase, in the liver and adipose tissue [6-10]. Moreover, ChREBP-Mlx regulates the glucose-induced expression of glucose-6-phosphate dehydrogenase $(G 6 P D H)$, the rate-limiting enzyme of the pentose phosphate pathway (PPP) [8].

The physiological function and key metabolic target genes of ChREBP/Mondo-Mlx are well conserved in animals and studies in Drosophila have revealed new insight into the physiological roles and tissue specificity of intracellular sugar sensing. Whereas the vertebrate genome encodes for two Mondo proteins, the fruit fly, Drosophila, has only one Mondo protein, displaying high expression in the fat body (corresponding to liver and adipose tissue), midgut (corresponding to small intestine) and Malpighian tubules (corresponding to kidney) [2]. Mondo-Mlx mediates a substantial proportion of Drosophila sugar-induced transcription and it activates conserved genes involved in glycolysis, lipogenesis, and the PPP, which is the most strongly over-represented 
Mondo-Mlx target pathway in Drosophila [11]. The PPP has a key role in producing NADPH reductive power, which is needed for the biosynthesis of lipids. Indeed, inhibition of the Mondo-Mlx induced activation of glucose-6-phosphate dehydrogenase leads to a dramatic reduction of TAG stores in Drosophila larvae [11]. The PPP is also essential for sugar tolerance. The reductive power of NADPH generated by the PPP prevents oxidative damage upon high sugar feeding through the glutathione system, which contributes to the animal's ability to tolerate a high sugar diet $[11,12]$.

In addition to conserved metabolic pathways, studies in Drosophila have revealed novel tissue-specific functions for sugar sensing. Mondo-Mlx is essential for the sugarinduced regulation of digestive enzymes in the midgut. Sugar feeding represses the expression of Amylases and Maltases, which are needed for digestion of complex carbohydrates $[13,14]$. This mechanism might have a role in regulating glucose intake, in order to prevent glucose overload [15]. Mondo-Mlx also regulates Malpighian tubule-specific glucose transporters, possibly in order to control excretion of excess glucose [11]. Furthermore, Mondo-Mlx is essential for normal flight muscle function and structure. Muscle-specific knockdown leads to accumulation of glycogen stores, possibly reflecting reduced glucose utilization [16]. In conclusion, ChREBP/MondoMlx is a conserved sugar sensor within animals, with essential roles in sugar-induced lipogenesis and coordinating whole body glucose homeostasis by controlling carbohydrate digestion and transport.

\section{ChREBP/Mondo-Mlx controls a gene regulatory network}

In addition to direct regulation of key metabolic genes Mondo-Mlx is emerging as a master regulator of other regulatory genes, including transcription factors (TFs) (Figure 1). Mondo-Mlx directly regulates Krüppel-like factor 10 (KLF10, also known as TIEG1) and its Drosophila ortholog Cabut. In Drosophila, the cabut promoter has two high affinity ChoREs. cabut is the most strongly downregulated gene in mlx mutants and depletion of Cabut by RNAi induces sugar intolerance in Drosophila larvae [2]. Similarly, in rat primary hepatocytes KLF10 expression is activated by glucose stimulation and hampered by dominant negative Mlx [17]. Elevated KLF10 expression is also observed in vivo in mouse following feeding on a high fat/high sugar diet [18]. Adenoviral overexpression of KLF10 in primary hepatocytes suppressed the glucoseinduced activation of ChREBP and several of its target genes, suggesting that KLF10 
120 can act as a negative feedback regulator of ChREBP [17]. A different outcome was

121 observed in loss-of-function experiments in Drosophila, where Cabut mediated

122 downregulation of a gene set in response to sugar feeding [19]. Among the most

123 strongly repressed Cabut target genes was phosphoenolpyruvate carboxykinase

124 (pepck), which is a direct Cabut target and also a target of KLF10 in mouse [19,20].

125 PEPCK is the rate-limiting enzyme of cataplerotic flux from the TCA cycle to mediate

126 channeling of carbon towards gluco- and glyceroneogenesis. Consistent with high

127 expression of pepck, Drosophila mlx mutants display high levels of glycerol and

128 trehalose (a circulating form of glucose in insects), which could be suppressed by

129 simultaneous loss of pepck [19]. Interestingly, Cabut also links sugar sensing to

130 regulation of circadian rhythm. Deregulation of Cabut has a dramatic impact on the

131 cycling of circadian metabolic gene output, while having no impact on the cycling of

132 core clock genes [19]. Notably, in rat hepatocytes ChREBP-Mlx also directly regulates

133 BHLHB2/DEC1, which has a well-established role in circadian regulation [21,22]. In

134 conclusion, through direct regulation of downstream transcription factors,

135 ChREBP/Mondo-Mlx-mediated sugar sensing appears to be closely coupled to the

136 circadian clock. Considering the observations on re-setting of the peripheral circadian

137 clock through timed feeding [23], it will be interesting to further explore the

138 physiological importance of the observed interplay between these TFs.

140 ChREBP/Mondo-Mlx also controls the expression of TFs involved in lipid 141 homeostasis. The Gli-similar (GLIS) transcription factor Sugarbabe has been long 142 known as the earliest and strongest sugar-responsive gene in Drosophila [14]. Recently, 143 sugarbabe was found to be a direct target of Mondo-Mlx. Loss- and gain of function 144 analyses revealed that Sugarbabe contributes to the lipid homeostasis in vivo. It 145 regulates fatty acid synthesis downstream of Mondo-Mlx by inducing the expression of 146 lipogenic genes $A C C, F A S, A c C o A S$ and $A T P C L$ in response to sugar feeding [11]. Gli147 similar transcription factor 2, the closest human homologue of Sugarbabe, was 148 originally identified as a bifunctional transcription factor, regulating both the activation 149 and repression of its target genes involved in kidney development [24]. Interestingly, 150 variants of another sugarbabe homolog, GLIS3, are now established as one of the 151 strongest known genetic risk factors for both type 1 and type 2 diabetes [25]. In addition 152 to direct regulation of other TFs, ChREBP-Mlx also regulates downstream effectors 153 indirectly. One such example is the fatty acid responsive nuclear receptor PPAR $\gamma$ [26]. 
154 ChREBP expression is elevated during adipocyte differentiation and ChREBP activity 155 is needed to maintain maximal transcriptional output of adipogenic PPAR $\gamma$. 156 Consequently, ectopic activation of ChREBP promotes adipocyte differentiation.

157 ChREBP likely affects PPAR $\gamma$ indirectly through lipid biosynthesis as the activating 158 effects of ChREBP depend on functional Fatty acid synthase [26]. In conclusion, 159 ChREBP/Mondo-Mlx contributes to lipid homeostasis through its direct targets as well 160 as through secondary TFs.

161

162 New hormonal targets of ChREBP/Mondo-Mlx

163 In addition to downstream transcription factors, ChREBP/Mondo-Mlx contributes to 164 systemic regulation through hormonal signals, including FGF21. In cultured 165 hepatocytes ChREBP-Mlx regulates the expression of FGF21 in a glucose-dependent 166 manner [8,27]. Levels of circulating FGF21 are rapidly increased in response to 167 fructose in humans as well as mice [28,29]. Interestingly, both baseline and fructose168 stimulated levels of FGF21 are significantly elevated in subjects with metabolic 169 syndrome [28]. This is consistent with findings showing elevated activation of hepatic 170 ChREBP in obese individuals [30]. Loss of FGF21 in turn leads to impaired de novo 171 lipogenesis in mouse liver, which is accompanied by liver fibrosis following prolonged 172 fructose exposure [29]. In addition to metabolic regulation, FGF21 also regulates sugar 173 feeding. Mice lacking FGF21 consume elevated levels of sucrose, whereas ectopic 174 FGF21 suppresses the intake of sugar in mice as well as cynomolgus monkeys $[31,32]$. 175 The liver-derived FGF21 suppresses sugar feeding in mice by acting through the FGF21 176 receptor complex in the paraventricular nucleus of the hypothalamus [31]. A similar 177 regulatory system likely exists in humans, since variants of $F G F 21$ are strongly 178 associated with sweet preference, as judged by the consumption of candy [33].

180 Research in Drosophila has revealed new connections between Mondo-Mlx and 181 hormonal metabolic regulation via TGF- $\beta$ /Activin signaling. TGF- $\beta /$ Activin ligand 182 Dawdle is highly expressed in the Drosophila fat body, from where it is secreted [15]. 183 Sugar feeding strongly elevates dawdle expression, while loss of $m l x$ leads to impaired 184 dawdle activation [11,15]. Moreover, Mondo-Mlx directly binds to a ChoRE at the 185 dawdle promoter [11]. Dawdle is essential in metabolic regulation as mutants of dawdle 186 show elevated circulating trehalose and glucose as well as high levels of glycogen and 
triacylglycerol [34]. Dawdle acts through multiple target tissues, for example it acts on

188 Drosophila insulin producing cells to promote insulin-like peptide secretion [34].

189 Moreover, Dawdle activates its receptor Baboon in intestinal enterocytes and 190 suppresses the expression of genes encoding carbohydrate digestive enzymes, 191 Amylases and Maltases, thus inhibiting carbohydrate digestion in response to excess 192 systemic glucose [15]. Interestingly, Dawdle functionally cooperates with the Mondo193 Mlx target TF Sugarbabe, which also contributes to the sugar-induced repression of 194 amylase expression [11]. In conclusion, studies in mammals and Drosophila have 195 opened new insight into the role of ChREBP/Mondo-Mlx on the systemic regulation of 196 metabolism via hormones secreted by the liver and fat body, respectively.

\section{Direct regulation of ChREBP/Mondo-Mlx by nutrient-derived cues}

199 ChREBP/Mondo TFs respond to sugars via their N-terminal Glucose sensing module 200 (GSM), which can be functionally divided into low-glucose inhibitory domain (LID) 201 and glucose response activation conserved element (GRACE) [35]. Although direct 202 structural evidence about ChREBP/Mondo activation mechanism is still missing, ample 203 indirect evidence suggests that direct binding of G6P, and possibly other 204 phosphorylated hexoses, lead to loss of intramolecular inhibition of GRACE by LID 205 [36,37]. Subsequently activated ChREBP/Mondo-Mlx dissociates from 14-3-3, 206 translocates to the nucleus, and binds to the ChoRE to activate its target genes (Figure

207 2A) [38-40]. Furthermore, glucose-responsive dephosphorylation as well as acetylation 208 have also been shown to contribute to ChREBP activation [41,42]. A detailed 209 representation of ChREBP/Mondo-Mlx activation has been presented elsewhere [3]. 210 This core activation mechanism allows ChREBP/Mondo-Mlx to control gene 211 expression in response to sugar availability. However, additional regulatory 212 mechanisms and functional interplay with other nutrient-responsive TFs to integrate 213 other nutrient-derived signals are beginning to emerge. One such signaling mechanism 214 is O-GlcNacylation. The synthesis of the substrate for O-GlcNacylation, UDP-GlcNAc, 215 through the hexosamine biosynthesis pathway (HBP), depends on availability of 216 nutrients, such as glucose and glutamine [43]. Thereby, O-GlcNacylation is considered 217 as a nutrient sensing mechanism. ChREBP is O-GlcNac modified [44-46] and recent 218 mass-spectrometric analysis have revealed multiple target sites [47]. Consistent with 219 the glucose sensitivity of the HBP, the levels of ChREBP O-GlcNac modification are 220 increased upon exposure to high glucose [44,45]. O-GlcNac modification increases 
221 ChREBP protein stability and increases the expression of ChREBP target genes [44].

222 Pancreatic $\beta$-cells have an inherent glucose sensing capacity and ChREBP activation

223 seems to involve a distinct mechanism in this setting. ChREBP forms a cytoplasmic

224 complex with a Calcium binding protein Sorcin [48]. Calcium influx in response to

225 high glucose releases ChREBP allowing its nuclear localization. In conclusion,

226 ChREBP activity is regulated by sugars through multiple parallel mechanisms.

228 In addition to posttranslational modifications, ChREBP/Mondo-Mlx is also regulated 229 at the transcriptional level. In mammals, the ChREBP gene encodes two isoforms, 230 ChREBP- $\alpha$ and ChREBP- $\beta$, which are transcribed from different promoters [49].

231 ChREBP- $\alpha$ is contains the N-terminal GSM and is thus directly activated by G6P.

232 ChREBP- $\alpha$ stimulates the expression of the shorter ChREBP- $\beta$, which lacks inherent 233 glucose sensing ability, but has a 20 -fold higher transcriptional activity than ChREBP-

$234 \alpha$. The promoter of ChREBP contains a binding site for Liver X receptor (LXR), and it 235 has been shown that LXR regulates the expression of ChREBP in the liver [50,51]. 236 However, the effect of LXR on ChREBP expression appears to be independent of 237 nutrient as the expression of ChREBP is not altered in high-sugar fed LXR mutant mice 238 [52]. Hepatocyte nuclear factor 4 alpha $(\mathrm{HNF}-4 \alpha)$ in turn has been shown to activate 239 the expression of both ChREBP isoforms in response to glucose [53]. Moreover, HNF$2404 \alpha$ has been also shown to physically interact with ChREBP, and this interaction is 241 promoted by glucose [53,54].

243 While ChREBP is activated in the liver of animals on a high sugar diet, a high lipid diet 244 has an inhibitory effect. Furthermore, administration of fatty acids inhibits nuclear 245 translocation and activation of ChREBP (Figure 2B) [55,56]. Fatty acids are oxidized 246 into ketone bodies, such as $\beta$-hydroxybutyrate and acetoacetate. By using an in vitro 247 binding assay with purified ChREBP and 14-3-3 as well as protein-free hepatocyte 248 extracts, Nagakawa and coworkers showed that $\beta$-hydroxybutyrate and acetoacetate 249 increase the binding between ChREBP and 14-3-3 [57]. This increased binding anchors 250 ChREBP to the cytoplasm, preventing its target gene activation. Fatty acid 251 administration also significantly elevates the levels of intracellular AMP [55]. AMP 252 activates the AMP-activated protein kinase (AMPK), which phosphorylates ChREBP 253 on Ser568 and inhibits its transcriptional activity, while having no impact on the nuclear 
254 localization [55,58]. Interestingly, a recent study suggests that AMP can also inhibit 255 ChREBP directly, through an allosteric mechanism. AMP binds to ChREBP and 256 increases its affinity to 14-3-3, which prevents ChREBP nuclear translocation [58]. In 257 conclusion, recent studies suggest that ChREBP acts as a direct sensor for several fatty 258 acid-derived metabolites, thus integrating multiple nutrient-derived cues.

\section{Cross-talk between ChREBP and other nutrient sensing pathways}

261 In addition to nutrient sensing through direct allosteric regulation and posttranslational 262 mechanisms, ChREBP/Mondo acts in close synergy with other nutrient sensing 263 systems, including insulin signaling. A well-established example is SREBP, a target of 264 insulin signaling, which controls synergistically lipogenic gene expression with 265 ChREBP [59]. In Drosophila, Mondo was shown to act synergistically with Salt266 inducible kinase 3 (SIK3), which is also a target of the insulin signaling pathway 267 [12,60]. Mondo and SIK3 act cooperatively to activate the PPP upon sugar feeding [12].

268 Similarly to Mondo, SIK3 is essential for sugar tolerance and animals with reduced 269 expression of both Mondo and SIK3 display extremely low tolerance towards dietary 270 sugars [12]. Moreover, ChREBP O-GlcNacylation is inhibited by Foxo1, which is an 271 inhibitory target of insulin signaling, thus providing additional cross-talk between 272 insulin-mediated systemic glucose homeostasis and intracellular glucose sensing [46].

273 ChREBP also likely modulates insulin signaling, since genome-wide analysis of

274 ChREBP chromatin binding in mouse liver and adipose tissue revealed significant 275 enrichment in ChREBP binding near genes encoding components of the insulin 276 signaling pathway [61].

278 Evidence about cross-talk between sugar sensing and amino acid sensing is also 279 emerging (Figure 2C). Glutamine is a critical metabolic intermediate, acting as a carbon 280 and ammonium carrier. Glutaminolysis is critical in replenishing the TCA cycle, when 281 TCA cycle carbon is channeled to biosynthesis. Interestingly, glutamine modifies 282 glucose sensing by inhibiting the transcriptional output of MondoA [62]. Consequently, 283 glutamine inhibits the glucose-induced activation of TXNIP, a negative regulator of 284 glucose transporter GLUT1 [63], thus increasing cellular glucose uptake [62]. 285 Glutamine has no impact on MondoA nuclear localization or DNA binding, but it likely 286 impacts MondoA cofactors [62]. MondoA can also control the uptake of glutamine. In 287 endothelial cells infected with Kaposi's Sarcoma-associated Herpesvirus, glutamine 
transporter SLC1A5 is activated in a MondoA-Mlx-dependent manner to facilitate glutaminolysis [64]. In conclusion, MondoA coordinates the intracellular balance of

290 glucose and glutamine catabolism.

292 The mechanistic target of rapamycin (mTOR) is a conserved intracellular amino acid 293 sensing system, which also has been shown to modulate the output of ChREBP/Mondo294 Mlx. mTOR inhibitors increase the expression of MondoA-Mlx target gene TXNIP 295 [65]. mTOR physically interacts with MondoA and sequesters it from forming an active 296 complex with Mlx [65]. Similarly to MondoA, ChREBP is inhibited by mTOR through 297 protein-protein interaction [66]. mTOR inhibition leads to increased expression of 298 TXNIP, which causes death of pancreatic $\beta$-cells [66]. Interestingly, the levels of 299 TXNIP are strongly elevated in diabetic islets, further suggesting that retaining the 300 interaction between cellular amino acid and glucose sensing is essential for normal $\beta$ 301 cell homeostasis. Recent evidence from C. elegans implies that Mondo-Mlx has a 302 capacity to modulate mTOR signaling, since loss of C. elegans homolog of Mondo and 303 Myc, MLL-1, leads to mTOR activation [67]. MLL-1 represses the expression of 304 leucyl-tRNA synthetase lars-1, whose activity promotes mTOR activation via RAG305 GTPases $[67,68]$. Through the regulation of mTOR signaling, MLL-1 is essential for 306 promoting longevity [67]. In sum, mTOR and ChREBP/Mondo pathways appear to be 307 closely interconnected, which potentially provides cross-coordination between 308 phenotypic outputs of dietary sugars and amino acids.

\section{Concluding remarks}

311 To conclude, recent work in the field has revealed that ChREBP/Mondo-Mlx regulates 312 a large regulatory network, including transcription factors and hormones, which 313 controls not only metabolic pathways, but also feeding activity and intestinal digestion.

314 In addition to intracellular sugar sensing, ChREBP/Mondo-Mlx integrates multiple 315 nutrient-responsive inputs, placing it at the very heart of an interlinked regulatory 316 system mediating physiological re-adjustment in response to nutrient intake. Such 317 integrated control allows animals to flourish in highly variable nutrient landscapes.

319 However, several questions remain to be addressed. These include elucidating the 320 structural basis of the function of the Glucose sensing module. This would allow better 
321 understanding of the "nutrient sensor" mechanism of ChREBP/Mondo and possibly to 322 enable design of new pharmaceuticals to modulate its activity. Given the availability of 323 animal models, understanding of the physiological roles of ChREBP/Mondo in 324 different tissues and cell types will certainly arise. Moreover, a wider perspective is 325 needed to understand the natural variation in dietary sugar intake, and the variation

326 between species and individuals with respect to their healthy nutrient landscape. As 327 ChREBP/Mondo-Mlx is a key determinant in sugar tolerance in mice and Drosophila, 328 it will be interesting to analyze its function in animals with differential sugar intake 329 and perhaps differential inherent sugar tolerance. Understanding the genetic factors 330 predisposing humans to disease on a high sugar diet would allow disease prevention 331 through personalized nutrition.

332

\section{Acknowledgements}

334 We thank Ryan Giblin and Mari Teesalu for feedback. Our research is supported by 335 Academy of Finland (grant no. 286767 to V.H.), Sigrid Juselius Foundation (to VH), 336 Novo Nordisk Foundation (NNF16OC0021460 to V.H.), Helsinki Institute of Life 337 Science (to VH), and the Finnish Diabetes Research Foundation (to V.H.). 


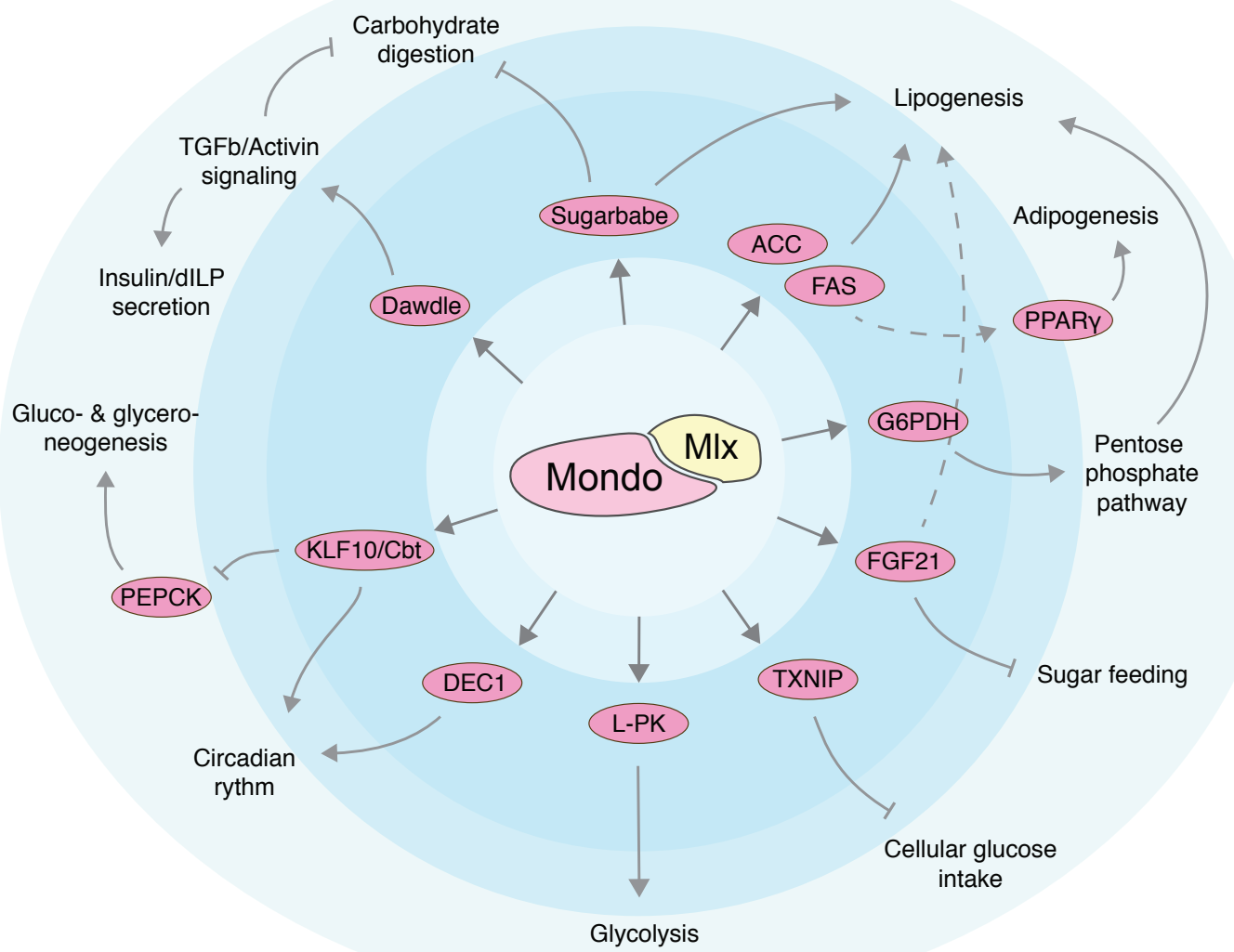

360 Figure 1. ChREBP/Mondo-Mlx is a master regulator of a sugar-induced gene

361 regulatory network. In response to sugars, ChREBP/Mondo-Mlx activates the 362 expression of a number of metabolic genes, but also other regulatory genes involved in 363 the control of carbohydrate and lipid metabolism, sugar feeding and circadian rhythm. 364 The figure summarizes key targets of mammalian ChREBP and MondoA as well as 365 Drosophila Mondo. Abbreviations: ACC: acetyl-CoA carboxylase, Cbt: Cabut, DEC1: 366 deleted in esophageal cancer 1, FAS: fatty acid synthase, FGF21: fibroblast growth 367 factor 21, G6PDH: glucose-6-phosphate dehydrogenase, KLF10: krüppel-like factor 368 10, L-PK: L-type pyruvate kinase, PEPCK: phosphoenolpyruvate carboxykinase, 369 PPAR $\gamma$ : peroxisome proliferator-activated receptor gamma, TXNIP: thioredoxin370 interacting protein. 

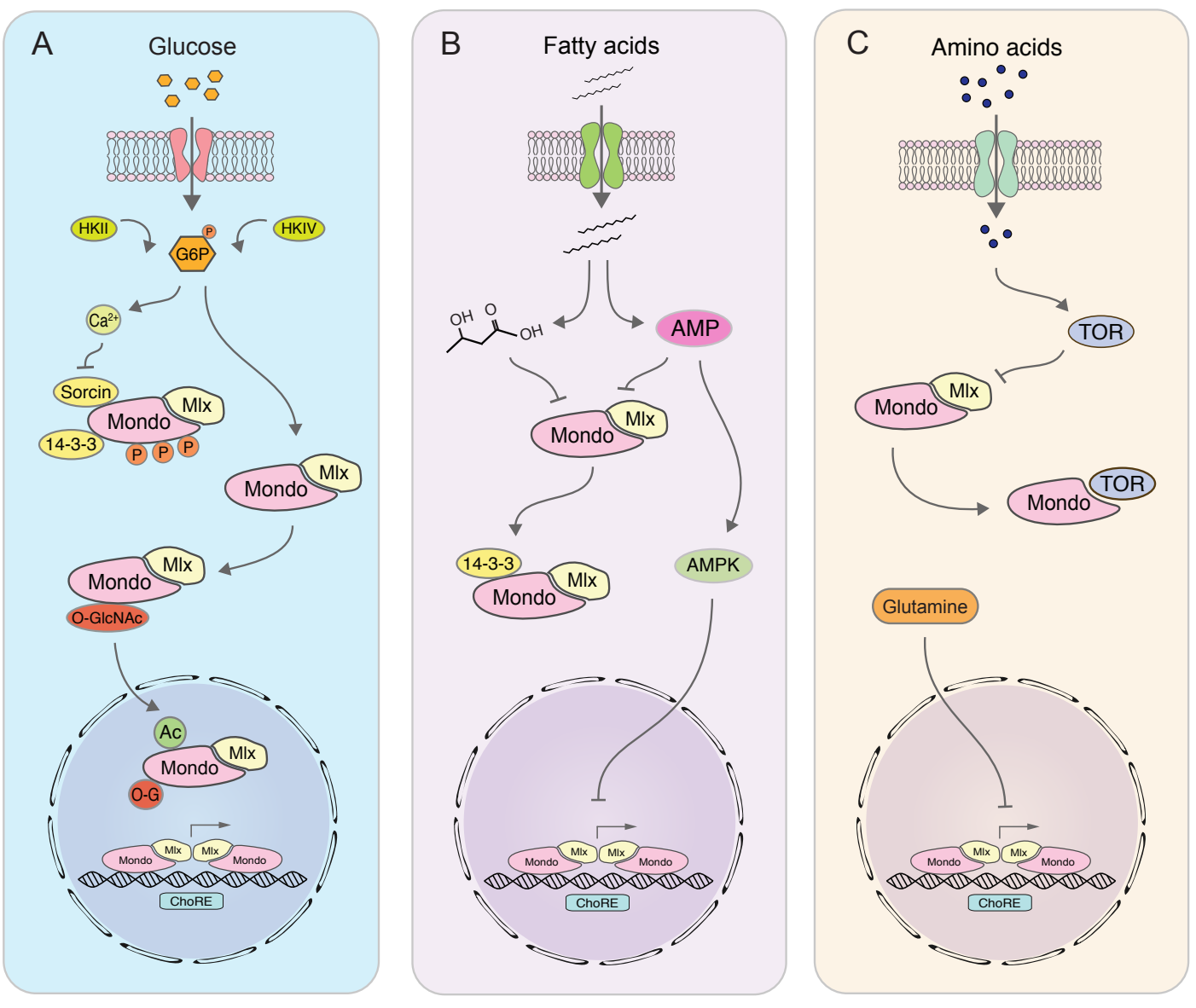

Figure 2. ChREBP/Mondo-MIx activity is regulated by many nutrient-derived cues. ChREBP/Mondo-Mlx is activated by glucose-6-phosphate (G6P) (A) and inhibited both by ketone bodies and AMP, whose levels depend on fatty acids (B). Direct binding of TOR to MondoA and ChREBP inhibits their function and glutamine inhibits the transcriptional activity of ChREBP-Mlx (C).

\section{Selected references}

\section{Of special interest *}

11. Mattila J, Havula E, Suominen E, Teesalu M, Surakka I, Hynynen R, Kilpinen H, Vaananen J, Hovatta I, Kakela R, et al.: Mondo-Mlx Mediates Organismal Sugar Sensing through the Gli-Similar Transcription Factor Sugarbabe. Cell Rep 2015, 13:350-364.

Genome-wide analysis of Mondo-Mlx regulated genes in Drosophila reveals Glisimilar TF Sugarbabe and Activin ligand Dawdle as direct Mondo-Mlx targets. 
26. Witte N, Muenzner M, Rietscher J, Knauer M, Heidenreich S, Nuotio-Antar AM, Graef FA, Fedders R, Tolkachov A, Goehring I, et al.: The Glucose Sensor ChREBP Links De Novo Lipogenesis to PPARgamma Activity and Adipocyte Differentiation. Endocrinology 2015, 156:4008-4019.

Reveals the role of ChREBP in adipocyte differentiation through indirect control of PPARgamma via lipogenesis.

32. Talukdar S, Owen BM, Song P, Hernandez G, Zhang Y, Zhou Y, Scott WT, Paratala B, Turner T, Smith A, et al.: FGF21 Regulates Sweet and Alcohol Preference. Cell Metab 2016, 23:344-349.

Shows that FGF21 inhibits sugar feeding in primates, in addition to mice.

61. Poungvarin N, Chang B, Imamura M, Chen J, Moolsuwan K, Sae-Lee C, Li W, Chan L: Genome-Wide Analysis of ChREBP Binding Sites on Male Mouse Liver and White Adipose Chromatin. Endocrinology 2015, 156:1982-1994.

Genomewide targets of ChREBP in liver and adipose tissues suggests ChREBPmediated control of the insulin signalling pathway. A valuable resource for future studies.

\section{Of outstanding interest **}

31. von Holstein-Rathlou S, BonDurant LD, Peltekian L, Naber MC, Yin TC, Claflin KE, Urizar AI, Madsen AN, Ratner C, Holst B, et al.: FGF21 Mediates Endocrine Control of Simple Sugar Intake and Sweet Taste Preference by the Liver. Cell Metab 2016, 23:335-343.

Uncovers that FGF21, whose expression is activated in the liver by ChREBP, inhibits feeding of sugars.

58. Sato S, Jung H, Nakagawa T, Pawlosky R, Takeshima T, Lee WR, Sakiyama H, Laxman S, Wynn RM, Tu BP, et al.: Metabolite Regulation of Nuclear Localization of Carbohydrate-response Element-binding Protein (ChREBP): ROLE OF AMP AS AN ALLOSTERIC INHIBITOR. $J$ Biol Chem 2016, 291:10515-10527.

Shows biochemical and structural evidence for AMP as a novel ligand to inhibit ChREBP nuclear localization by enhancing the interaction between ChREBP and 143-3.

65. Kaadige MR, Yang J, Wilde BR, Ayer DE: MondoA-Mlx transcriptional activity is limited by mTOR-MondoA interaction. Mol Cell Biol 2015, 35:101-110.

Demonstrates direct interaction between mTOR and MondoA to inhibit MondoA activation. 
67. Nakamura S, Karalay O, Jager PS, Horikawa M, Klein C, Nakamura K, Latza C, Templer SE, Dieterich C, Antebi A: Mondo complexes regulate TFEB via TOR inhibition to promote longevity in response to gonadal signals. Nat Commun 2016, 7:10944.

Demonstrates Mondo-mediated regulation of TOR activity and its impact for longevity in C. elegans.

\section{References}

1. Iizuka K, Bruick RK, Liang G, Horton JD, Uyeda K: Deficiency of carbohydrate response element-binding protein (ChREBP) reduces lipogenesis as well as glycolysis. Proc Natl Acad Sci U S A 2004, 101:7281-7286.

2. Havula E, Teesalu M, Hyotylainen T, Seppala H, Hasygar K, Auvinen P, Oresic M, Sandmann $\mathrm{T}$, Hietakangas V: Mondo/ChREBP-Mlx-Regulated Transcriptional Network Is Essential for Dietary Sugar Tolerance in Drosophila. PLoS Genet 2013, 9:e1003438.

3. Havula E, Hietakangas V: Glucose sensing by ChREBP/MondoA-Mlx transcription factors. Semin Cell Dev Biol 2012.

4. Sans CL, Satterwhite DJ, Stoltzman CA, Breen KT, Ayer DE: MondoA-MIx heterodimers are candidate sensors of cellular energy status: mitochondrial localization and direct regulation of glycolysis. Mol Cell Biol 2006, 26:4863-4871.

5. Billin AN, Eilers AL, Coulter KL, Logan JS, Ayer DE: MondoA, a novel basic helix-loop-helix-leucine zipper transcriptional activator that constitutes a positive branch of a max-like network. Mol Cell Biol 2000, 20:8845-8854.

6. Ishii S, Iizuka K, Miller BC, Uyeda K: Carbohydrate response element binding protein directly promotes lipogenic enzyme gene transcription. Proc Natl Acad Sci U S A 2004, 101:15597-15602.

7. Jeong YS, Kim D, Lee YS, Kim HJ, Han JY, Im SS, Chong HK, Kwon JK, Cho YH, Kim WK, et al.: Integrated expression profiling and genome-wide analysis of ChREBP targets reveals the dual role for ChREBP in glucose-regulated gene expression. PLoS One 2011, 6:e22544.

8. Ma L, Robinson LN, Towle HC: ChREBP*Mlx is the principal mediator of glucose-induced gene expression in the liver. J Biol Chem 2006, 281:2872128730.

9. Ma L, Tsatsos NG, Towle HC: Direct role of ChREBP.Mlx in regulating hepatic glucose-responsive genes. $J$ Biol Chem 2005, 280:12019-12027.

10. Yamashita H, Takenoshita M, Sakurai M, Bruick RK, Henzel WJ, Shillinglaw W, Arnot D, Uyeda K: A glucose-responsive transcription factor that regulates carbohydrate metabolism in the liver. Proc Natl Acad Sci U S 2001, 98:9116-9121.

11. Mattila J, Havula E, Suominen E, Teesalu M, Surakka I, Hynynen R, Kilpinen H, Vaananen J, Hovatta I, Kakela R, et al.: Mondo-Mlx Mediates Organismal Sugar Sensing through the Gli-Similar Transcription Factor Sugarbabe. Cell Rep 2015, 13:350-364.

12. Teesalu M, Rovenko BM, Hietakangas V: Salt-Inducible Kinase 3 Provides Sugar Tolerance by Regulating NADPH/NADP+ Redox Balance. Curr Biol 2017, 27:458-464. 
13. Benkel BF, Hickey DA: Glucose Repression of Amylase Gene Expression in DROSOPHILA MELANOGASTER. Genetics 1986, 114:137-144.

14. Zinke I, Schutz CS, Katzenberger JD, Bauer M, Pankratz MJ: Nutrient control of gene expression in Drosophila: microarray analysis of starvation and sugar-dependent response. $E M B O J$ 2002, 21:6162-6173.

15. Chng WB, Bou Sleiman MS, Schupfer F, Lemaitre B: Transforming growth factor beta/activin signaling functions as a sugar-sensing feedback loop to regulate digestive enzyme expression. Cell Rep 2014, 9:336-348.

16. Polak GL, Pasqualino A, Docherty JE, Beck SJ, DiAngelo JR: The Regulation of Muscle Structure and Metabolism by Mio/dChREBP in Drosophila. PLoS One 2015, 10:e0136504.

17. Iizuka $\mathrm{K}$, Takeda $\mathrm{J}$, Horikawa $\mathrm{Y}$ : Kruppel-like factor-10 is directly regulated by carbohydrate response element-binding protein in rat primary hepatocytes. Biochem Biophys Res Commun 2011, 412:638-643.

18. Kim JK, Lee KS, Chang HY, Lee WK, Lee JI: Progression of diet induced nonalcoholic steatohepatitis is accompanied by increased expression of Kruppel-like-factor 10 in mice. J Transl Med 2014, 12:186.

19. Bartok O, Teesalu M, Ashwall-Fluss R, Pandey V, Hanan M, Rovenko BM, Poukkula M, Havula E, Moussaieff A, Vodala S, et al.: The transcription factor Cabut coordinates energy metabolism and the circadian clock in response to sugar sensing. $E M B O J$ 2015, 34:1538-1553.

20. Guillaumond F, Grechez-Cassiau A, Subramaniam M, Brangolo S, Peteri-Brunback B, Staels B, Fievet C, Spelsberg TC, Delaunay F, Teboul M: Kruppel-like factor KLF10 is a link between the circadian clock and metabolism in liver. Mol Cell Biol 2010, 30:3059-3070.

21. Iizuka K, Horikawa Y: Regulation of lipogenesis via BHLHB2/DEC1 and ChREBP feedback looping. Biochem Biophys Res Commun 2008, 374:95-100.

22. Honma S, Kawamoto T, Takagi Y, Fujimoto K, Sato F, Noshiro M, Kato Y, Honma K: Dec1 and Dec2 are regulators of the mammalian molecular clock. Nature 2002, 419:841-844.

23. Damiola F, Le Minh N, Preitner N, Kornmann B, Fleury-Olela F, Schibler U: Restricted feeding uncouples circadian oscillators in peripheral tissues from the central pacemaker in the suprachiasmatic nucleus. Genes Dev 2000, 14:2950-2961.

24. Zhang F, Nakanishi G, Kurebayashi S, Yoshino K, Perantoni A, Kim YS, Jetten AM: Characterization of Glis2, a novel gene encoding a Gli-related, Kruppel-like transcription factor with transactivation and repressor functions. Roles in kidney development and neurogenesis. $J$ Biol Chem 2002, 277:10139-10149.

25. Yang Y, Chan L: Monogenic Diabetes: What It Teaches Us on the Common Forms of Type 1 and Type 2 Diabetes. Endocr Rev 2016, 37:190-222.

26. Witte N, Muenzner M, Rietscher J, Knauer M, Heidenreich S, Nuotio-Antar AM, Graef FA, Fedders R, Tolkachov A, Goehring I, et al.: The Glucose Sensor ChREBP Links De Novo Lipogenesis to PPARgamma Activity and Adipocyte Differentiation. Endocrinology 2015, 156:4008-4019.

27. Iizuka K, Takeda J, Horikawa Y: Glucose induces FGF21 mRNA expression through ChREBP activation in rat hepatocytes. FEBS Lett 2009, 583:28822886. 
28. Dushay JR, Toschi E, Mitten EK, Fisher FM, Herman MA, Maratos-Flier E: Fructose ingestion acutely stimulates circulating FGF21 levels in humans. Mol Metab 2015, 4:51-57.

29. Fisher FM, Kim M, Doridot L, Cunniff JC, Parker TS, Levine DM, Hellerstein MK, Hudgins LC, Maratos-Flier E, Herman MA: A critical role for ChREBPmediated FGF21 secretion in hepatic fructose metabolism. Mol Metab 2017, 6:14-21.

30. Eissing L, Scherer T, Todter K, Knippschild U, Greve JW, Buurman WA, Pinnschmidt HO, Rensen SS, Wolf AM, Bartelt A, et al.: De novo lipogenesis in human fat and liver is linked to ChREBP-beta and metabolic health. Nat Commun 2013, 4:1528.

31. von Holstein-Rathlou S, BonDurant LD, Peltekian L, Naber MC, Yin TC, Claflin KE, Urizar AI, Madsen AN, Ratner C, Holst B, et al.: FGF21 Mediates Endocrine Control of Simple Sugar Intake and Sweet Taste Preference by the Liver. Cell Metab 2016, 23:335-343.

32. Talukdar S, Owen BM, Song P, Hernandez G, Zhang Y, Zhou Y, Scott WT, Paratala B, Turner T, Smith A, et al.: FGF21 Regulates Sweet and Alcohol Preference. Cell Metab 2016, 23:344-349.

33. Soberg S, Sandholt CH, Jespersen NZ, Toft U, Madsen AL, von Holstein-Rathlou S, Grevengoed TJ, Christensen KB, Bredie WLP, Potthoff MJ, et al.: FGF21 Is a Sugar-Induced Hormone Associated with Sweet Intake and Preference in Humans. Cell Metab 2017, 25:1045-1053 e1046.

34. Ghosh AC, O'Connor MB: Systemic Activin signaling independently regulates sugar homeostasis, cellular metabolism, and $\mathbf{p H}$ balance in Drosophila melanogaster. Proc Natl Acad Sci U S A 2014, 111:5729-5734.

35. Li MV, Chang B, Imamura M, Poungvarin N, Chan L: Glucose-dependent transcriptional regulation by an evolutionarily conserved glucose-sensing module. Diabetes 2006, 55:1179-1189.

36. Davies MN, O'Callaghan BL, Towle HC: Activation and repression of glucosestimulated ChREBP requires the concerted action of multiple domains within the MondoA conserved region. Am J Physiol Endocrinol Metab 2010, 299:E665-674.

37. McFerrin LG, Atchley WR: Evolution of the Max and Mlx networks in animals. Genome Biol Evol 2011, 3:915-937.

38. Sakiyama H, Wynn RM, Lee WR, Fukasawa M, Mizuguchi H, Gardner KH, Repa JJ, Uyeda K: Regulation of nuclear import/export of carbohydrate response element-binding protein (ChREBP): interaction of an alpha-helix of ChREBP with the 14-3-3 proteins and regulation by phosphorylation. $J$ Biol Chem 2008, 283:24899-24908.

39. Eilers AL, Sundwall E, Lin M, Sullivan AA, Ayer DE: A novel heterodimerization domain, CRM1, and 14-3-3 control subcellular localization of the MondoAMlx heterocomplex. Mol Cell Biol 2002, 22:8514-8526.

40. Merla G, Howald C, Antonarakis SE, Reymond A: The subcellular localization of the ChoRE-binding protein, encoded by the Williams-Beuren syndrome critical region gene 14, is regulated by 14-3-3. Hum Mol Genet 2004, 13:1505-1514.

41. Kabashima T, Kawaguchi T, Wadzinski BE, Uyeda K: Xylulose 5-phosphate mediates glucose-induced lipogenesis by xylulose 5-phosphate-activated protein phosphatase in rat liver. Proc Natl Acad Sci U S A 2003, 100:51075112. 
42. Bricambert J, Miranda J, Benhamed F, Girard J, Postic C, Dentin R: Salt-inducible kinase 2 links transcriptional coactivator p300 phosphorylation to the prevention of ChREBP-dependent hepatic steatosis in mice. $J$ Clin Invest 2010, 120:4316-4331.

43. Hanover JA, Krause MW, Love DC: The hexosamine signaling pathway: OGIcNAc cycling in feast or famine. Biochim Biophys Acta 2010, 1800:80-95.

44. Guinez C, Filhoulaud G, Rayah-Benhamed F, Marmier S, Dubuquoy C, Dentin R, Moldes M, Burnol AF, Yang X, Lefebvre T, et al.: O-GlcNAcylation increases ChREBP protein content and transcriptional activity in the liver. Diabetes 2011, 60:1399-1413.

45. Park MJ, Kim DI, Lim SK, Choi JH, Han HJ, Yoon KC, Park SH: High glucoseinduced $\mathrm{O}-$ GlcNAcylated carbohydrate response element-binding protein (ChREBP) mediates mesangial cell lipogenesis and fibrosis: the possible role in the development of diabetic nephropathy. J Biol Chem 2014, 289:13519-13530.

46. Ido-Kitamura Y, Sasaki T, Kobayashi M, Kim HJ, Lee YS, Kikuchi O, YokotaHashimoto H, Iizuka K, Accili D, Kitamura T: Hepatic FoxO1 integrates glucose utilization and lipid synthesis through regulation of Chrebp Oglycosylation. PLoS One 2012, 7:e47231.

47. Yang AQ, Li D, Chi L, Ye XS: Validation, Identification, and Biological Consequences of the Site-specific O-GIcNAcylation Dynamics of Carbohydrate-responsive Element-binding Protein (ChREBP). Mol Cell Proteomics 2017, 16:1233-1243.

48. Noordeen NA, Meur G, Rutter GA, Leclerc I: Glucose-induced nuclear shuttling of ChREBP is mediated by sorcin and $\mathrm{Ca}(2+)$ ions in pancreatic beta-cells. Diabetes 2012, 61:574-585.

49. Herman MA, Peroni OD, Villoria J, Schon MR, Abumrad NA, Bluher M, Klein S, Kahn BB: A novel ChREBP isoform in adipose tissue regulates systemic glucose metabolism. Nature 2012, 484:333-338.

50. Cha JY, Repa JJ: The liver $X$ receptor (LXR) and hepatic lipogenesis. The carbohydrate-response element-binding protein is a target gene of LXR. $J$ Biol Chem 2007, 282:743-751.

51. Gauthier K, Billon C, Bissler M, Beylot M, Lobaccaro JM, Vanacker JM, Samarut J: Thyroid hormone receptor beta (TRbeta) and liver $X$ receptor (LXR) regulate carbohydrate-response element-binding protein (ChREBP) expression in a tissue-selective manner. J Biol Chem 2010, 285:28156-28163.

52. Denechaud PD, Bossard P, Lobaccaro JM, Millatt L, Staels B, Girard J, Postic C: ChREBP, but not LXRs, is required for the induction of glucose-regulated genes in mouse liver. $J$ Clin Invest 2008, 118:956-964.

53. Meng J, Feng M, Dong W, Zhu Y, Li Y, Zhang P, Wu L, Li M, Lu Y, Chen H, et al.: Identification of HNF-4alpha as a key transcription factor to promote ChREBP expression in response to glucose. Sci Rep 2016, 6:23944.

54. Adamson AW, Suchankova G, Rufo C, Nakamura MT, Teran-Garcia M, Clarke $\mathrm{SD}$, Gettys TW: Hepatocyte nuclear factor-4alpha contributes to carbohydrate-induced transcriptional activation of hepatic fatty acid synthase. Biochem J 2006, 399:285-295.

55. Kawaguchi T, Osatomi K, Yamashita H, Kabashima T, Uyeda K: Mechanism for fatty acid "sparing" effect on glucose-induced transcription: regulation of carbohydrate-responsive element-binding protein by AMP-activated protein kinase. J Biol Chem 2002, 277:3829-3835. 
56. Dentin R, Benhamed F, Pegorier JP, Foufelle F, Viollet B, Vaulont S, Girard J, Postic C: Polyunsaturated fatty acids suppress glycolytic and lipogenic genes through the inhibition of ChREBP nuclear protein translocation. $J$ Clin Invest 2005, 115:2843-2854.

57. Nakagawa T, Ge Q, Pawlosky R, Wynn RM, Veech RL, Uyeda K: Metabolite regulation of nucleo-cytosolic trafficking of carbohydrate response element-binding protein (ChREBP): role of ketone bodies. $J$ Biol Chem 2013, 288:28358-28367.

58. Sato S, Jung H, Nakagawa T, Pawlosky R, Takeshima T, Lee WR, Sakiyama H, Laxman S, Wynn RM, Tu BP, et al.: Metabolite Regulation of Nuclear Localization of Carbohydrate-response Element-binding Protein (ChREBP): ROLE OF AMP AS AN ALLOSTERIC INHIBITOR. $J$ Biol Chem 2016, 291:10515-10527.

59. Dentin R, Girard J, Postic C: Carbohydrate responsive element binding protein (ChREBP) and sterol regulatory element binding protein-1c (SREBP-1c): two key regulators of glucose metabolism and lipid synthesis in liver. Biochimie 2005, 87:81-86.

60. Wang B, Moya N, Niessen S, Hoover H, Mihaylova MM, Shaw RJ, Yates JR, 3rd, Fischer WH, Thomas JB, Montminy M: A hormone-dependent module regulating energy balance. Cell 2011, 145:596-606.

61. Poungvarin N, Chang B, Imamura M, Chen J, Moolsuwan K, Sae-Lee C, Li W, Chan L: Genome-Wide Analysis of ChREBP Binding Sites on Male Mouse Liver and White Adipose Chromatin. Endocrinology 2015, 156:1982-1994.

62. Kaadige MR, Looper RE, Kamalanaadhan S, Ayer DE: Glutamine-dependent anapleurosis dictates glucose uptake and cell growth by regulating MondoA transcriptional activity. Proc Natl Acad Sci U S A 2009, 106:1487814883.

63. Wu N, Zheng B, Shaywitz A, Dagon Y, Tower C, Bellinger G, Shen CH, Wen J, Asara J, McGraw TE, et al.: AMPK-dependent degradation of TXNIP upon energy stress leads to enhanced glucose uptake via GLUT1. Mol Cell 2013, 49:1167-1175.

64. Sanchez EL, Carroll PA, Thalhofer AB, Lagunoff M: Latent KSHV Infected Endothelial Cells Are Glutamine Addicted and Require Glutaminolysis for Survival. PLoS Pathog 2015, 11:e1005052.

65. Kaadige MR, Yang J, Wilde BR, Ayer DE: MondoA-MIx transcriptional activity is limited by mTOR-MondoA interaction. Mol Cell Biol 2015, 35:101-110.

66. Chau GC, Im DU, Kang TM, Bae JM, Kim W, Pyo S, Moon EY, Um SH: mTOR controls ChREBP transcriptional activity and pancreatic beta cell survival under diabetic stress. $J$ Cell Biol 2017, 216:2091-2105.

67. Nakamura S, Karalay O, Jager PS, Horikawa M, Klein C, Nakamura K, Latza C, Templer SE, Dieterich $C$, Antebi A: Mondo complexes regulate TFEB via TOR inhibition to promote longevity in response to gonadal signals. Nat Commun 2016, 7:10944.

68. Han JM, Jeong SJ, Park MC, Kim G, Kwon NH, Kim HK, Ha SH, Ryu SH, Kim $\mathrm{S}$ : Leucyl-tRNA synthetase is an intracellular leucine sensor for the mTORC1-signaling pathway. Cell 2012, 149:410-424. 